Research Article

\title{
Diagnostic Fragment-Ion-Based for Rapid Identification of Chlorogenic Acids Derivatives in Inula cappa Using UHPLC-Q- Exactive Orbitrap Mass Spectrometry
}

\author{
Jie Peng $\mathbb{D}^{1},{ }^{1}$ Jing Xie $\mathbb{D},{ }^{2}$ Silin Shi $\mathbb{D},{ }^{1}$ Lilan Luo $\mathbb{D},{ }^{1}$ Kailin Li $\mathbb{D},{ }^{1}$ Pei Xiong $\mathbb{D},{ }^{1}$ \\ and Wei Cai ${ }^{1,3}$ \\ ${ }^{1}$ School of Pharmaceutical Sciences, Hunan Province Key Laboratory for Antibody-Based Drug and Intelligent Delivery System, \\ Hunan University of Medicine, Huaihua 418000, China \\ ${ }^{2}$ Department of Rehabilitation Medicine and Health Care of Hunan Medical College, Hunan University of Medicine, \\ Huaihua 418000, China \\ ${ }^{3}$ Hunan Provincial Key Laboratory of Dong Medicine, Hunan University of Medicine, Huaihua 418000, China
}

Correspondence should be addressed to Wei Cai; 20120941161@bucm.edu.cn

Received 30 May 2021; Accepted 13 August 2021; Published 23 August 2021

Academic Editor: Antony C. Calokerinos

Copyright (c 2021 Jie Peng et al. This is an open access article distributed under the Creative Commons Attribution License, which permits unrestricted use, distribution, and reproduction in any medium, provided the original work is properly cited.

\begin{abstract}
Inula cappa (Buch.-Ham. ex D. Don) DC has been used in traditional Chinese medicine to treat malaria, dysentery, and hepatitis. Previous studies have shown that chlorogenic acid is the effective ingredient of plants in this family. And the research of the chlorogenic acid in Inula cappa will help to further improve the effective resource utilization rate of this plant. Therefore, it is necessary to establish an accurate method to characterize the chlorogenic acid components in Inula cappa. In this study, a simple, fast, and sensitive UHPLC-Q-Exactive Orbitrap mass spectrometry method was established, which can simultaneously analyze known and unknown ingredients in a short time (within 30 minutes) in Inula cappa. According to the diagnosis fragmentation ions, retention time, and bibliography, 68 chlorogenic acid derivatives were identified in Inula cappa. The results of this experiment lay the foundation for the active substances and quality control of Inula cappa and provide a theoretical basis for whether Inula cappa can be an alternative to the endangered wild medicinal materials of the same family.
\end{abstract}

\section{Introduction}

Traditional Chinese medicine (TCM) has been applied for thousands of years in China and its surrounding areas. For a long time, TCM has attracted worldwide attention because of its extremely effective treatment of certain diseases and minimal side effects. It was estimated that more than 1.5 billion people all over the world trust in the efficacy of TCM and rest assured of its safety [1]. However, it is still unclear which of the ingredients of TCM are effective for the treatment of diseases because they are a complex mixture of hundreds of different chemical components. It not only hinders the clinical promotion and application of TCM but also greatly hinders the development of TCM. In recent years, with the continuous improvement and advancement of analytical techniques and methodology, the research speed of TCM has been greatly accelerated.
Based on the diversity and complexity of the chemical components of TCM, it is necessary to use advanced analytical techniques and methods to explain the pharmacological basis and mechanism of action of TCM because ultrahigh performance liquid chromatography-mass spectrometry (UHPLC-MS) with high sensitivity is to reduce sample complexity [2]. In addition, UHPLC-MS also has the advantages of good specificity, short analysis cycle, and good signal reproducibility $[3,4]$. UHPLC-HRMS has now been widely used in the analysis and identification of TCM components, especially most small molecules.

Inula cappa (Buch-Ham. ex D. Don) DC, a perennial shrub, belonging to the family composite, known as "Yang Er Ju" in Chinese, is widely distributed in the southern areas of China, such as Hunan, Sichuan, Yunnan, Guizhou, Guangxi, Guangdong, and Zhejiang provinces. Its roots or 
the whole plant have traditionally been used as medicines for treatment of malaria, dysentery, and hepatitis [4]. The compounds responsible for the various pharmacological effects of Inula cappa consist of sesquiterpene lactones (isoalantolactone and germacranolide), triterpenoids (lupeol, oleanolic acid, and $\beta$-sitosterol), steroids, anthraquinones, flavonoids (luteolin, apigenin, and chrysoeriol), fragrances, amides, and chlorogenic acid $[5,6]$.

Chlorogenic acids (CGAs) are the family of esters phytochemicals formed between cinnamic acid derivatives and (-)-quinic acids. In recent years, chlorogenic acid have been proven to mediate for its hepatoprotective, choleretic, antimicrobial activities, antioxidant, hypoglycaemic, and antiviral activities; besides, basic and clinical investigations have implied that the consumption of chlorogenic acid can reduce the relative risks of type 2 diabetes, obesity, and Alzheimer's disease [7, 8].

In this study, a simple, fast, and sensitive UHPLC-QExactive Orbitrap mass spectrometry method was established, based on accurate mass, which can simultaneously analyze the known and unknown components in Inula cappa in a short time (within 30 minutes).

First, extracting the CGAs from the Inula cappa by refluxing, a high-resolution mass spectrum was obtained by using UHPLC-Q-Exactive Orbitrap MS in the negative mode and parallel reaction monitoring. Finally, the diagnostic fragment ions, retention time, and bibliography were established to process the ion chromatograms, and 68 compounds in the Inula cappa were rapidly identified.

\section{Materials and Methods}

2.1. Chemicals and Reagents. HPLC grade of methanol, acetonitrile, and formic acid were purchased from Fisher scientific Company (New jersey, USA). Standards substances of the following phytochemicals were purchased from Chengdu Herbpurify Co., Ltd. (Chengdu, China): trans-3-caffeoylquinic acid (neochlorogenic acid, X-014-170309), trans-4-caffeoylquinic acid (cryptochlorogenic acid, Y-067-180320), trans-5caffeoylquinic acid (chlorogenic acid, L-007-171216), 3,5dicaffeoylquinic acid (isochlorogenic acid A, Y-068-170903), 3,4-dicaffeoylquinic acid (isochlorogenic acid B, Y-069180105), 4,5-dicaffeoylquinic acid (isochlorogenic acid C, Y-070-170515), 1,3-dicaffeo-pyridinequinic acid (cimarin, MUST-16022610), and 1,5-dicaffeo-pyridinequinic acid (cimarin, MUST-16022610).

2.2. Materials. The decoction pieces of Inula cappa (dried root) were purchased from Bozhou Jianzheng Trading Co., Ltd., (Henan, China), which were grounded into powder before sample preparation. The voucher specimen was deposited at School of Pharmaceutical Sciences, Hunan University of Medicine.

2.3. Instrumentation. A Q-Exactive Focus Orbitrap MS (Thermo Electron, Bremen, Germany) was connected to the Thermo Scientific Dionex Ultimate 3000 RS (Thermo Fisher Scientific, California, USA) via an ESI source. A UPW-N
Series Water Purification System was purchased from LeiCi, Shanghai Yidian Scientific Instrument Co., Ltd., Shanghai, China. An automatic dual range professional type of analytical balances PTX-FA210 was purchased from Huazhi Electronic Technology Co., Ltd., Fujian, China.

2.4. Preparation of Standard Solutions. Accurately weigh $10 \mathrm{mg}$ of each reference standard, and dissolve it in $10 \mathrm{~mL}$ of methanol; then, take $10 \mu \mathrm{L}$ and dilute to $1 \mathrm{~mL}$. A volume of $1 \mu \mathrm{L}$ was injected into UHPLC-Q-Exactive Orbitrap MS for analysis.

2.5. Preparation of Sample Solutions. The dried powder of Inula cappa $(10 \mathrm{~g})$ was reflux-extracted in $50 \mathrm{~mL} 70 \%$ aqueous ethanol for $1 \mathrm{~h}$, and then, the extracted solution was filtered and dried by rotary evaporation and then reconstituted with $10 \mathrm{ml}$ methanol. A volume of $2 \mu \mathrm{L}$ was injected into UHPLC-Q-Exactive Orbitrap MS for analysis.

2.6. HPLC Chromatographic Condition. An Hypersil GOLD aQ $(100 \mathrm{~mm} \times 2.1 \mathrm{~mm}, 1.9 \mu \mathrm{m})$ was used for chromatographic separation at $35^{\circ} \mathrm{C}$. The mobile phase consisted of $0.1 \%$ formic acid (A) and acetonitrile (B) at a flow rate of $0.3 \mathrm{~mL} / \mathrm{min}$ in the following gradient: $0 \mathrm{~min}, 5 \% \mathrm{~B} ; 2 \mathrm{~min}$, $10 \% \mathrm{~B} ; 5 \mathrm{~min}, 20 \% \mathrm{~B} ; 10 \mathrm{~min}, 25 \% \mathrm{~B} ; 12 \mathrm{~min}, 55 \% \mathrm{~B} ; 20 \mathrm{~min}$, $80 \% \mathrm{~B} ; 25 \mathrm{~min}, 95 \% \mathrm{~B} ; 26 \mathrm{~min}, 5 \% \mathrm{~B}$; and $30 \mathrm{~min}, 5 \% \mathrm{~B}$.

All samples were analyzed in the negative mode as the following tune method. The nitrogen (purity $\geq 99.99 \%$ ) served as sheath gas and auxiliary gas at the flow rate of 30 and 10 (arbitrary unit), respectively; the capillary temperature is $320^{\circ} \mathrm{C}$; the auxiliary gas heater temperature is $350^{\circ} \mathrm{C}$; and spray voltage is $3.5 \mathrm{kV}$. High-resolution mass spectrum was acquired at full scan in a mass range of m/z 120-1000 at a resolution of 35000 detected by the Orbitrap analyzer. The $\mathrm{MS}^{2}$ data at a resolution of 17500 were obtained by the parallel reaction monitoring mode triggered by the inclusion ions list. The nitrogen (purity $\geq 99.999 \%$ ) served as collision gas to generate the fragment ions, and the energy was set as normalized collision energy $30 \%$ [9].

2.7. The Establishment of Diagnosis Fragmentation Ions. Chlorogenic acid (CGA) is a series of ester phytochemicals formed between caffeic acid and quinic acid. It is easy to understand that all CGAs use quinic acid as the backbone and produce similar fragments, which can be defined as diagnostic ions. By using diagnostic ion information, CGAs can be quickly screened and characterized. The fragmentations behavior of CGAs has been reported and summarized in the previous literature.

For example, for CGAs with the same quinic acid as the backbone of quinine, cinnamoyl residues (such as caffeoyl and ferulic acid esters) are usually cleaved and lost on the ester bond, thus yielding about 191.0552 at $\mathrm{m} / \mathrm{z}$, and the characteristic product ion corresponds to [quinic acid- $\mathrm{H}$ ]$\left(\mathrm{C}_{7} \mathrm{H}_{12} \mathrm{O}_{6}\right)$. The diagnostic ions at $\mathrm{m} / \mathrm{z} 353.0875$ and $\mathrm{m} / \mathrm{z}$ 515.1195 correspond to mono-CQA and diCQA $\mathrm{m} / \mathrm{z}$ 191.0561 and $\mathrm{m} / \mathrm{z} 173.0455$ are present in all types of chlorogenic acid and can be used as diagnostic ions. The ions 
with mass numbers m/z 173 and m/z 193 derived from dehydroquinic acid and ferulic acid are used as diagnostic ions, which can diagnose FQA (especially 4FQA) and CQA at the same time [10].

2.8. Data Processing and Analysis. LC-MS data analysis was performed using Xcalibur software version 4 (Thermo Fisher Scientific, San Jose, California, USA). The raw data including the full-scan MS and $\mathrm{MS}^{2}$ data were processed by the Compound Discover 3.0 using the expected compounds predicted method [11] based on the metabolism workflow templates to detect the chlorogenic acid derivatives constituents of Inula cappa. Finally, candidates for CGA were characterized based on the diagnostic fragment ions, retention time, and bibliography.

\section{Result}

The table lists all the chlorogenic acid and its derivatives detected in the extracted Inula cappa sample by UHPLC-QExactive Orbitrap mass spectrometry based on diagnostic fragment ions, retention time, and bibliographical identification (Table 1). A total of 68 chlorogenic acids and their derivatives were identified (Figure 1).

3.1. Identification of Monoacyl-Quinic Acids or MonoacylShikimic Acids. Compounds 14, 19, 22, 24, 28, 30, 34, and 41 generated the same quasimolecular ions $[\mathrm{M}-\mathrm{H}]^{-}$at $\mathrm{m} / \mathrm{z}$ $335.076\left(\mathrm{C}_{16} \mathrm{H}_{15} \mathrm{O}_{8}\right)$ which eluted at $4.63,5.15,5.65,5.81$, $6.14,6.38,6.86$, and $8.32 \mathrm{~min}$, respectively. According to previously reported literature, these compounds may be either caffeoylquinic acid lactones (CQL) or caffeoylshikimic acids (CSA). Due to the loss of lactone and $\mathrm{H}_{2} \mathrm{O}$ moiety, quinic acid lactones prone to generate ions at $\mathrm{m} / \mathrm{z} 161.023$, so $\mathrm{m} / \mathrm{z} 161.023$ can be used to distinguish CQLs and CSAs. For the above reasons, these compounds were tentatively identified as 3-CQL, 1-CQL, 4-CQL, 4-CSA, 3-CSA, 1-CQL, 4-CQL, and 1-CQL, respectively [12-15].

Compounds 23, 26, and 32 were eluted at 5.76, 5.97, and $6.71 \mathrm{~min}$ and showed a deprotonated molecular ion $[\mathrm{M}-\mathrm{H}]^{-}$ at $\mathrm{m} / \mathrm{z} \quad 337.09299 \quad\left(1.20 \mathrm{ppm}, \quad \mathrm{C}_{16} \mathrm{H}_{17} \mathrm{O}_{8}\right), \quad 337.09320$ (1.41 ppm, $\mathrm{C}_{16} \mathrm{H}_{17} \mathrm{O}_{8}$ ), and $337.09351\left(1.71 \mathrm{ppm}, \mathrm{C}_{16} \mathrm{H}_{17} \mathrm{O}_{8}\right.$ ), respectively. According to previous literature analysis, the base peak ions and fragment ions of the $\mathrm{MS}^{2}$ spectrum were temporarily designated as 5-O-p-coumaroylquinic acid (pCoQA), 5-pCoQA, and 1-pCoQA [16].

Compound 6 was eluted at $3.76 \mathrm{~min}$, with the deprotonated ion $[\mathrm{M}-\mathrm{H}]^{-}$at $\mathrm{m} / \mathrm{z} \quad 499.14609 \quad(1.47 \mathrm{ppm}$, $\left.\mathrm{C}_{22} \mathrm{H}_{27} \mathrm{O}_{13}\right), \quad 162 \mathrm{Da}\left(\mathrm{C}_{6} \mathrm{H}_{10} \mathrm{O}_{5}\right)$ more than pCoQA $\left(\mathrm{C}_{16} \mathrm{H}_{17} \mathrm{O}_{8}\right)$. The fragment ion generated at $\mathrm{m} / \mathrm{z} 337.09$ $\left(\mathrm{C}_{16} \mathrm{H}_{17} \mathrm{O}_{8}\right)$ by loss the $\mathrm{C}_{6} \mathrm{H}_{10} \mathrm{O}_{5}$ moiety was detected in $\mathrm{MS}^{2}$ spectrum of compound 6 , indicating that it was hexoside of pCoQA. The fragment ions at $\mathrm{m} / \mathrm{z} 173.044$ and 191.054 were shown in the $\mathrm{MS}^{2}$ spectrum of compound 6, indicating that compound 6 was 4-pCo, 5CQA, respectively [12-14].

Compounds 2, 4, and 18 were eluted at 2.85, 3.33, and $4.89 \mathrm{~min}$ and generated the same deprotonated ion $[\mathrm{M}-\mathrm{H}]^{-}$ at $\mathrm{m} / \mathrm{z} 353.08\left(\mathrm{C}_{16} \mathrm{H}_{17} \mathrm{O}_{9}\right)$. According to comparing the retention time, MS data with those reference standards, compounds 2,4 , and 18 were accurately characterized as cis3-CQA, trans-3-CQA, and trans-5-CQA [17].

Compounds 1, 3, 7, 10, and 13 eluted at 2.71, 3.28, 3.76, 4.00 , and $4.50 \mathrm{~min}$ and showed a deprotonated molecular ion $[\mathrm{M}-\mathrm{H}]^{-}$at $\mathrm{m} / \mathrm{z} 515.14050\left(-0.25 \mathrm{ppm}, \mathrm{C}_{22} \mathrm{H}_{27} \mathrm{O}_{14}\right)$, $515.13959\left(-2.02 \mathrm{ppm}, \mathrm{C}_{22} \mathrm{H}_{27} \mathrm{O}_{14}\right), 515.14069$ (0.12 ppm, $\left.\mathrm{C}_{22} \mathrm{H}_{27} \mathrm{O}_{14}\right), \quad 515.14081 \quad\left(0.35 \mathrm{ppm}, \mathrm{C}_{22} \mathrm{H}_{27} \mathrm{O}_{14}\right)$, and $515.14105\left(0.82 \mathrm{ppm}, \mathrm{C}_{22} \mathrm{H}_{27} \mathrm{O}_{14}\right), 162 \mathrm{Da}\left(\mathrm{C}_{6} \mathrm{H}_{10} \mathrm{O}_{5}\right)$ more than CQA, indicating they were the hexoside of CQA. The presence of fragment ion at $\mathrm{m} / \mathrm{z} 323.076\left(\mathrm{C}_{15} \mathrm{H}_{15} \mathrm{O}_{8}\right)$ can distinguish the attachment sites of hexosides. In summary, 1 , 3 , and 7 are tentatively designated as CQA $-4^{\prime}$-hexoside, and the others may be CQA- $3^{\prime}$-hexoside $[12,14,18]$.

Compounds 16, 29, 31, and 53 were eluted at 4.85, 6.23, 6.42 , and $11.30 \mathrm{~min}$ and generated deprotonated ions [M$\mathrm{H}]^{-}$at $\mathrm{m} / \mathrm{z} 367.10333\left(0.97 \mathrm{ppm}, \mathrm{C}_{17} \mathrm{H}_{19} \mathrm{O}_{9}\right), 367.10352$ (1.16 ppm, $\mathrm{C}_{17} \mathrm{H}_{19} \mathrm{O}_{9}$ ), $367.10352\left(1.16 \mathrm{ppm}, \mathrm{C}_{17} \mathrm{H}_{19} \mathrm{O}_{9}\right.$ ), and 367.10199 ( $-3.99 \mathrm{ppm}, \mathrm{C}_{17} \mathrm{H}_{19} \mathrm{O}_{9}$ ), respectively, indicating they were the feruloylquinic acid (FQA). According to the base peak ion generated at $\mathrm{m} / \mathrm{z} 193.049\left(\mathrm{C}_{10} \mathrm{H}_{9} \mathrm{O}_{4}\right)$, it was determined to be 3-FQA. The base peak ion generated at $\mathrm{m} / \mathrm{z}$ $173.044\left(\mathrm{C}_{7} \mathrm{H}_{9} \mathrm{O}_{5}\right)$ was determined to be $4 \mathrm{FQA}$; the base peak ion generated at $\mathrm{m} / \mathrm{z} 191.054\left(\mathrm{C}_{7} \mathrm{H}_{11} \mathrm{O}_{6}\right)$ was determined to be 5FQA. Above all, they were tentatively characterized as 3FQA, 4FQA, 5FQA, and 4FQA $[16,17]$.

Compounds 5 and 12 with the deprotonated ions [M$\mathrm{H}]^{-}$at $\mathrm{m} / \mathrm{z} 529.15628 \quad\left(-2.02 \mathrm{ppm}, \mathrm{C}_{23} \mathrm{H}_{29} \mathrm{O}_{14}\right)$ and $529.15649\left(0.40 \mathrm{ppm}, \mathrm{C}_{23} \mathrm{H}_{29} \mathrm{O}_{14}\right)$ were eluted at 3.33 and $4.39 \mathrm{~min}, 162 \mathrm{Da}\left(\mathrm{C}_{6} \mathrm{H}_{10} \mathrm{O}_{5}\right)$ more than FQA, indicating they were the hexoside of FQA. The presence of fragment ions $\mathrm{m} /$ $z$ 193.049, 173.044, and 367.102 further confirmed the above identification. The base peak ion of $\mathrm{m} / \mathrm{z} 529$ can be used to distinguish the submitted position of the above groups. In summary, compound 5 was tentatively identified as 3-FQAhexosides, and 12 is 4FQA-hexosides [12-14].

3.2. Identification of Diacyl-Quinic Acids or Diacyl-Shikimic Acids. Compounds 51, 55, and 56 possessed deprotonated ions $[\mathrm{M}-\mathrm{H}]^{-}$at $\mathrm{m} / \mathrm{z} 497.10956\left(1.72 \mathrm{ppm}, \mathrm{C}_{25} \mathrm{H}_{21} \mathrm{O}_{11}\right)$, 497.10944 (1.60 ppm, $\mathrm{C}_{25} \mathrm{H}_{21} \mathrm{O}_{11}$ ), and 497.10950 (1,66 ppm, $\mathrm{C}_{25} \mathrm{H}_{21} \mathrm{O}_{11}$ ) and were eluted at $11.20,11.68$, and $12.24 \mathrm{~min}$, which could be dicaffeoylquinic acid lactones (DiCQL) or dicaffeoylshikimic acids (DiCSA). Compounds 51, 55, and 56 had the same base peak at m/z 161.0230 by the loss of lactone and $\mathrm{H}_{2} \mathrm{O}$ part of quinine lactone, so they were tentatively named DICQL [12-14].

Compounds $36,37,43,45,52$, and 67 were eluted at 7.05 , $7.23,9.22,9.72,11.26$, and $13.41 \mathrm{~min}$ and showed the deprotonated ion $[\mathrm{M}-\mathrm{H}]^{-}$at $\mathrm{m} / \mathrm{z} 499.12653$ (3.89 ppm, $\left.\mathrm{C}_{25} \mathrm{H}_{23} \mathrm{O}_{11}\right), 499.12396\left(0.47 \mathrm{ppm}, \mathrm{C}_{25} \mathrm{H}_{23} \mathrm{O}_{11}\right), 499.12460$ (1.11 ppm, $\left.\mathrm{C}_{25} \mathrm{H}_{23} \mathrm{O}_{11}\right), 499.12515$ (1.66 ppm, $\mathrm{C}_{25} \mathrm{H}_{23} \mathrm{O}_{11}$ ), $499.12451 \quad\left(1.02 \mathrm{ppm}, \quad \mathrm{C}_{25} \mathrm{H}_{23} \mathrm{O}_{11}\right)$, and 499.12097 $\left(-2.52 \mathrm{ppm}, \mathrm{C}_{25} \mathrm{H}_{23} \mathrm{O}_{11}\right)$, respectively. In the $\mathrm{MS}^{2}$ spectra of these compounds, the characteristic fragment ions $\mathrm{m} / \mathrm{z}$ $173.044 \quad\left(\mathrm{C}_{7} \mathrm{H}_{9} \mathrm{O}_{5}\right), \quad 179.033 \quad\left(\mathrm{C}_{9} \mathrm{H}_{7} \mathrm{O}_{4}\right)$, and 191.054 $\left(\mathrm{C}_{7} \mathrm{H}_{11} \mathrm{O}_{6}\right)$ of p-coumaroyl-caffeoylquinic acids (pCoCQA) were appeared, indicating that they were pCoCQA. 
TABle 1: The retention time and mass spectrometric data of CGAs in Inula cappa.

\begin{tabular}{|c|c|c|c|c|c|c|c|}
\hline Peak & $t_{R}$ & $\begin{array}{l}\text { Theoretical } \\
\text { mass }(\mathrm{m} / \mathrm{z})\end{array}$ & $\begin{array}{l}\text { Experimental } \\
\text { mass }(\mathrm{m} / \mathrm{z})\end{array}$ & $\begin{array}{l}\text { Error } \\
(\mathrm{ppm})\end{array}$ & $\begin{array}{c}\text { Formula } \\
{[\mathrm{M}-\mathrm{H}]}\end{array}$ & MS/MS fragment & $\begin{array}{l}\text { Identification/ } \\
\text { reactions }\end{array}$ \\
\hline 1 & 2.71 & 515.14063 & 515.14050 & -0.25 & $\mathrm{C}_{22} \mathrm{H}_{27} \mathrm{O}_{14}$ & $\mathrm{MS}^{2}$ [515]: 179.0343 (100), 191.0555 (21) & $\begin{array}{l}\text { CQA-4'- } \\
\text { hexoside }\end{array}$ \\
\hline 2 & 2.85 & 353.08781 & 353.08798 & 1.27 & $\mathrm{C}_{16} \mathrm{H}_{17} \mathrm{O}_{9}$ & $\operatorname{MS}^{2}[353]: 191.0554(100)$ & Cis-3-CQA \\
\hline 3 & 3.28 & 515.14063 & 515.13959 & -2.02 & $\mathrm{C}_{22} \mathrm{H}_{27} \mathrm{O}_{14}$ & $\begin{array}{c}\mathrm{MS}^{2}[515]: 179.0342(100), 191.0554(27) \\
341.0878(16)\end{array}$ & $\begin{array}{l}\text { CQA-4' } \\
\text { hexoside }\end{array}$ \\
\hline 4 & 3.33 & 353.08781 & 353.08789 & 1.18 & $\mathrm{C}_{16} \mathrm{H}_{17} \mathrm{O}_{9}$ & $\begin{array}{c}\mathrm{MS}^{2} \text { [353]: } 191.0554(100), 135.0440(82), \\
179.0341(45)\end{array}$ & Trans-3-CQA \\
\hline 5 & 3.33 & 529.15628 & 529.15521 & -2.02 & $\mathrm{C}_{23} \mathrm{H}_{29} \mathrm{O}_{14}$ & $\begin{array}{c}\mathrm{MS}^{2} \text { [529]: } 193.0499(100), 191.0554(35) \\
173.0447(25)\end{array}$ & 3-FQA-hexoside \\
\hline 6 & 3.76 & 499.14571 & 499.14609 & 1.47 & $\mathrm{C}_{22} \mathrm{H}_{27} \mathrm{O}_{13}$ & $\begin{array}{c}\mathrm{MS}^{2} \text { [499]: } 173.0447(100), 93.0333(80) \\
191.0555(24), 163.0392(14)\end{array}$ & 4-pCo, 5CQA \\
\hline 7 & 3.76 & 515.14063 & 515.14069 & 0.12 & $\mathrm{C}_{22} \mathrm{H}_{27} \mathrm{O}_{14}$ & $\mathrm{MS}^{2}[515]: 191.0555(100)$ & $\begin{array}{l}\text { CQA-4' - } \\
\text { hexoside }\end{array}$ \\
\hline 8 & 3.83 & 353.10894 & 353.10687 & -0.97 & $\mathrm{C}_{13} \mathrm{H}_{21} \mathrm{O}_{11}$ & $\begin{array}{c}\mathrm{MS}^{2} \text { [353]: } 191.0554(100), 85.02814(7) \\
173.0449(4)\end{array}$ & QA-hexoside \\
\hline 9 & 3.97 & 341.08781 & 341.08798 & 1.27 & $\mathrm{C}_{15} \mathrm{H}_{17} \mathrm{O}_{9}$ & $\mathrm{MS}^{2}$ [341]: 135.0441 (100), 179.0342 (59) & CA-hexoside \\
\hline 10 & 4.00 & 515.14063 & 515.14081 & 0.35 & $\mathrm{C}_{22} \mathrm{H}_{27} \mathrm{O}_{14}$ & $\begin{array}{c}\mathrm{MS}^{2}[515]: 173.0447(100), 179.0342(62) \\
191.0554(43), 353.0884(3)\end{array}$ & $\begin{array}{l}\text { CQA-3'- } \\
\text { hexoside }\end{array}$ \\
\hline 11 & 4.16 & 341.08781 & 341.08801 & 1.30 & $\mathrm{C}_{15} \mathrm{H}_{17} \mathrm{O}_{9}$ & $\begin{array}{c}\mathrm{MS}^{2}[341]: 135.0441(100), 179.0342(59), \\
161.0235(14)\end{array}$ & CA-hexoside \\
\hline 12 & 4.39 & 529.15628 & 529.15649 & 0.40 & $\mathrm{C}_{23} \mathrm{H}_{29} \mathrm{O}_{14}$ & $\begin{array}{c}\mathrm{MS}^{2} \text { [529]: } 173.0447(100), 191.0554(25) \\
111.0439(20), 193.0499(13)\end{array}$ & 4FQA-hexoside \\
\hline 13 & 4.50 & 515.14063 & 515.14105 & 0.82 & $\mathrm{C}_{22} \mathrm{H}_{27} \mathrm{O}_{14}$ & $\mathrm{MS}^{2}$ [515]: 191.0554 (100), 161.0235 (14) & $\begin{array}{l}\text { CQA-3'- } \\
\text { hexoside }\end{array}$ \\
\hline 14 & 4.63 & 335.07724 & 335.07733 & 1.19 & $\mathrm{C}_{16} \mathrm{H}_{15} \mathrm{O}_{8}$ & $\mathrm{MS}^{2}$ [335]: 161.0235 (100), 173.0600 (22) & 3-CQL \\
\hline 15 & 4.64 & 341.08781 & 341.08801 & 1.30 & $\mathrm{C}_{15} \mathrm{H}_{17} \mathrm{O}_{9}$ & 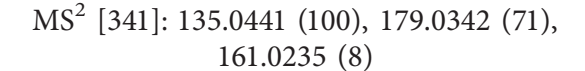 & CA-hexoside \\
\hline 16 & 4.85 & 367.10346 & 367.10333 & 0.97 & $\mathrm{C}_{17} \mathrm{H}_{19} \mathrm{O}_{9}$ & $\mathrm{MS}^{2}$ [367]: 134.0362 (100), 193.0499 (59) & 3-FQA \\
\hline 17 & 4.87 & 839.22515 & 839.22515 & 0.23 & $\mathrm{C}_{37} \mathrm{H}_{43} \mathrm{O}_{22}$ & $\begin{array}{c}\mathrm{MS}^{2} \text { [839]: } 353.0881(100), 191.0556(76), \\
173.0448(8)\end{array}$ & $\begin{array}{l}\text { DiCQA- } \\
\text { dihexoside }\end{array}$ \\
\hline 18 & 4.89 & 353.08781 & 353.08783 & 1.12 & $\mathrm{C}_{16} \mathrm{H}_{17} \mathrm{O}_{9}$ & $\begin{array}{c}\mathrm{MS}^{2} \text { [353]: } 191.0554(100), 135.0441(20), \\
173.0447(15), 179.0342(9)\end{array}$ & Trans-5-CQA \\
\hline 19 & 5.15 & 335.07724 & 335.07678 & -1.37 & $\mathrm{C}_{16} \mathrm{H}_{15} \mathrm{O}_{8}$ & $\begin{array}{c}\mathrm{MS}^{2}[335]: 161.0235(100), 201.0553(55), \\
59.0125(52), 173.0816(24)\end{array}$ & 1-CQL \\
\hline 20 & 5.34 & 839.22515 & 839.22595 & 0.96 & $\mathrm{C}_{37} \mathrm{H}_{43} \mathrm{O}_{22}$ & $\begin{array}{l}\mathrm{MS}^{2} \text { [839]: } 341.0878(100), 515.1416(76) \\
179.0342(32), 335.0775(16), 353.0884(5)\end{array}$ & $\begin{array}{l}\text { DiCQA- } \\
\text { dihexoside }\end{array}$ \\
\hline 21 & 5.56 & 677.17232 & 677.17285 & 0.78 & $\mathrm{C}_{31} \mathrm{H}_{33} \mathrm{O}_{17}$ & $\begin{array}{c}\mathrm{MS}^{2}[677]: 515.1409(100), 341.0879(87) \\
179.0342(64), 353.0879(32), 191.0553(18)\end{array}$ & $\begin{array}{l}\text { DiCQA- } \\
\text { hexoside }\end{array}$ \\
\hline 22 & 5.65 & 335.07724 & 335.07730 & 1.16 & $\mathrm{C}_{16} \mathrm{H}_{15} \mathrm{O}_{8}$ & $\begin{array}{c}\mathrm{MS}^{2}[335]: 161.0235(100), 135.0440(55) \\
179.0339(15), 173.04453(7)\end{array}$ & 4-CQL \\
\hline 23 & 5.76 & 337.09289 & 337.09299 & 1.20 & $\mathrm{C}_{16} \mathrm{H}_{17} \mathrm{O}_{8}$ & $\mathrm{MS}^{2}$ [337]: 173.0447 (100), 163.0392 (25) & 5-pCoQA \\
\hline 24 & 5.81 & 335.07724 & 335.07785 & 1.71 & $\mathrm{C}_{16} \mathrm{H}_{15} \mathrm{O}_{8}$ & $\mathrm{MS}^{2}$ [335]: 135.0441 (100), 179.0343 (31) & 4-CSA \\
\hline 25 & 5.88 & 677.17232 & 677.17468 & 3.48 & $\mathrm{C}_{31} \mathrm{H}_{33} \mathrm{O}_{17}$ & $\begin{array}{c}\mathrm{MS}^{2}[677]: 353.0880(100), 179.0342(92) \\
191.0555(48), \\
341.0878(30), 335.0775(25) \\
173.0447(10)\end{array}$ & $\begin{array}{l}\text { DiCQA- } \\
\text { hexoside }\end{array}$ \\
\hline 26 & 5.97 & 337.09289 & 337.09320 & 1.41 & $\mathrm{C}_{16} \mathrm{H}_{17} \mathrm{O}_{8}$ & $\mathrm{MS}^{2}$ [337]: 191.0554 (100), 163.0392 (15) & 5-pCoQA \\
\hline 27 & 6.08 & 515.11950 & 515.11969 & 1.29 & $\mathrm{C}_{25} \mathrm{H}_{23} \mathrm{O}_{12}$ & $\begin{array}{c}\mathrm{MS}^{2} \text { [515]: } 191.0554(100), 179.0342(95) \\
135.0441(14), 353.0880(14)\end{array}$ & 1,3-DiCQA \\
\hline 28 & 6.14 & 335.07724 & 335.07755 & 1.41 & $\mathrm{C}_{16} \mathrm{H}_{15} \mathrm{O}_{8}$ & $\begin{array}{c}\mathrm{MS}^{2}[335]: 135.0441(100), 161.0235(97), \\
173.0447(51), 179.0342(37)\end{array}$ & 3-CSA \\
\hline 29 & 6.23 & 367.10346 & 367.10352 & 1.16 & $\mathrm{C}_{17} \mathrm{H}_{19} \mathrm{O}_{9}$ & $\begin{array}{c}\mathrm{MS}^{2}[367]: 173.0447(100), 93.0333(33) \\
134.0362(32), 193.0500(13)\end{array}$ & 4FQA \\
\hline 30 & 6.38 & 335.07724 & 335.07727 & 1.13 & $\mathrm{C}_{16} \mathrm{H}_{15} \mathrm{O}_{8}$ & $\mathrm{MS}^{2}$ [335]: 161.0235 (100), 135.0441 (25) & 1-CQL \\
\hline 31 & 6.42 & 367.10346 & 367.10352 & 1.16 & $\mathrm{C}_{17} \mathrm{H}_{19} \mathrm{O}_{9}$ & $\begin{array}{c}\mathrm{MS}^{2}[367]: 191.0554(100), 93.0333(54) \\
134.0362(19), 173.0447(17), 193.0500(13)\end{array}$ & 5FQA \\
\hline 32 & 6.71 & 337.09289 & 337.09351 & 1.71 & $\mathrm{C}_{16} \mathrm{H}_{17} \mathrm{O}_{8}$ & $\begin{array}{c}\mathrm{MS}^{2} \text { [337]: } 191.0555(100), 173.0599(72) \\
163.0392(28), 119.0491(19)\end{array}$ & 1-pCoQA \\
\hline 33 & 6.73 & 341.08781 & 341.08786 & 0.16 & $\mathrm{C}_{15} \mathrm{H}_{17} \mathrm{O}_{9}$ & $\begin{array}{c}\mathrm{MS}^{2}[341]: 135.0441(100), 179.0343(18) \\
161.0599(16)\end{array}$ & CA-hexoside \\
\hline
\end{tabular}


TABle 1: Continued.

\begin{tabular}{|c|c|c|c|c|c|c|c|}
\hline Peak & $t_{R}$ & $\begin{array}{l}\text { Theoretical } \\
\text { mass }(\mathrm{m} / \mathrm{z})\end{array}$ & $\begin{array}{l}\text { Experimental } \\
\text { mass }(\mathrm{m} / \mathrm{z})\end{array}$ & $\begin{array}{l}\text { Error } \\
(\mathrm{ppm})\end{array}$ & $\begin{array}{c}\text { Formula } \\
{[\mathrm{M}-\mathrm{H}]}\end{array}$ & MS/MS fragment & $\begin{array}{l}\text { Identification/ } \\
\text { reactions }\end{array}$ \\
\hline 34 & 6.86 & 335.07724 & 335.07727 & 1.13 & $\mathrm{C}_{16} \mathrm{H}_{15} \mathrm{O}_{8}$ & $\begin{array}{c}\mathrm{MS}^{2} \text { [335]: } 161.0235(100), 173.1174(12), \\
135.0040(11)\end{array}$ & 4-CQL \\
\hline 35 & 6.93 & 529.13515 & 529.13611 & 1.82 & $\mathrm{C}_{26} \mathrm{H}_{25} \mathrm{O}_{12}$ & $\begin{array}{c}\mathrm{MS}^{2}[335]: 179.0342(100), 191.0554(91), \\
205.0324(30)\end{array}$ & 3C, 5FQA \\
\hline 36 & 7.05 & 499.12458 & 499.12653 & 3.89 & $\mathrm{C}_{25} \mathrm{H}_{23} \mathrm{O}_{11}$ & $\begin{array}{c}\mathrm{MS}^{2}[335]: 179.0342(100), 191.0554(91), \\
205.0324(30)\end{array}$ & 3C, 5-pCoQA \\
\hline 37 & 7.23 & 499.12458 & 499.12396 & 0.47 & $\mathrm{C}_{25} \mathrm{H}_{23} \mathrm{O}_{11}$ & $\begin{array}{l}\mathrm{MS}^{2}[499]: 163.0392(100), 191.0555(33), \\
173.0448(9), 179.0340(7), 337.09310(6)\end{array}$ & $\begin{array}{l}\text { Cis-3-pCo, } \\
\text { 5CQA }\end{array}$ \\
\hline 38 & 7.38 & 677.17232 & 677.17145 & -1.3 & $\mathrm{C}_{31} \mathrm{H}_{33} \mathrm{O}_{17}$ & $\begin{array}{c}\mathrm{MS}^{2}[677]: 353.0880(100), 191.0555(56) \\
515.1446(46), 179.0343(30), 341.0876(20) \\
173.0448(19)\end{array}$ & $\begin{array}{l}\text { DiCQA- } \\
\text { hexoside }\end{array}$ \\
\hline 39 & 8.02 & 515.11950 & 515.11938 & 0.98 & $\mathrm{C}_{25} \mathrm{H}_{23} \mathrm{O}_{12}$ & $\begin{array}{c}\mathrm{MS}^{2}[515]: 173.0447(100), 179.0342(92) \\
191.0554(38), 353.0879(15), 135.0441(14) \\
161.0235(14)\end{array}$ & 3,4-DiCQA \\
\hline 40 & 8.31 & 515.11950 & 515.11945 & 1.05 & $\mathrm{C}_{25} \mathrm{H}_{23} \mathrm{O}_{12}$ & $\begin{array}{c}\mathrm{MS}^{2}[515]: 191.0554(100), 179.0342(60) \\
353.0881(14), 135.0441(9)\end{array}$ & 3,5-DiCQA \\
\hline 41 & 8.32 & 335.07724 & 335.07709 & 0.95 & $\mathrm{C}_{16} \mathrm{H}_{15} \mathrm{O}_{8}$ & $\begin{array}{c}\mathrm{MS}^{2} \text { [335]: } 161.0235(100), 135.0441(95) \\
173.0447(46), 179.0342(31)\end{array}$ & 1-CQL \\
\hline 42 & 9.12 & 515.11950 & 515.11926 & 0.86 & $\mathrm{C}_{25} \mathrm{H}_{23} \mathrm{O}_{12}$ & $\begin{array}{c}\mathrm{MS}^{2}[515]: 173.0447(100), 179.0342(67) \\
191.0555(25), 353.0880(21), 135.0441(10)\end{array}$ & 4,5-DiCQA \\
\hline 43 & 9.22 & 499.12458 & 499.12460 & 1.11 & $\mathrm{C}_{25} \mathrm{H}_{23} \mathrm{O}_{11}$ & $\begin{array}{c}\mathrm{MS}^{2}[499]: 163.0392(100), 173.0447(84), \\
179.0342(65), 191.0555(31), 165.0912(29), \\
197.0449(24), 135.0440(16), 335.0776(11)\end{array}$ & $\begin{array}{l}\text { Cis-3-pCo, } \\
\text { 5CQA }\end{array}$ \\
\hline 44 & 9.54 & 529.13515 & 529.13525 & 0.19 & $\mathrm{C}_{26} \mathrm{H}_{25} \mathrm{O}_{12}$ & $\begin{array}{c}\mathrm{MS}^{2}[529]: 193.0499(100), 173.0447(73) \\
179.0342(62), 161.0235(17), 335.0777(16) \\
135.0440(14), 191.0554(12)\end{array}$ & 3F, 5CQA \\
\hline 45 & 9.72 & 499.12458 & 499.12515 & 1.66 & $\mathrm{C}_{25} \mathrm{H}_{23} \mathrm{O}_{11}$ & $\begin{array}{l}\mathrm{MS}^{2} \text { [499]: } 191.0555(100), 163.0392(39) \\
179.0343(33), 173.0447(13), 135.0441(8)\end{array}$ & 3C, 5-pCoQA \\
\hline 46 & 9.76 & 529.13515 & 529.13550 & 0.66 & $\mathrm{C}_{26} \mathrm{H}_{25} \mathrm{O}_{12}$ & $\begin{array}{c}\mathrm{MS}^{2} \text { [529]: } 173.0447(100), 193.0499(28), \\
179.0342(11)\end{array}$ & 3F, 5CQA \\
\hline 47 & 10.11 & 529.13515 & 529.13550 & 0.66 & $\mathrm{C}_{26} \mathrm{H}_{25} \mathrm{O}_{12}$ & $\mathrm{MS}^{2}$ [529]: 193.0499 (100), 173.0447 (14) & $3 \mathrm{~F}, 5 \mathrm{CQA}$ \\
\hline 48 & 10.28 & 529.13515 & 529.13550 & 0.66 & $\mathrm{C}_{26} \mathrm{H}_{25} \mathrm{O}_{12}$ & $\begin{array}{c}\mathrm{MS}^{2} \text { [529]: } 191.0554(100), 193.0499(61) \\
179.0341(33), 173.0447(19)\end{array}$ & 3C, 5FQA \\
\hline 49 & 10.32 & 677.15119 & 677.15173 & 0.79 & $\mathrm{C}_{34} \mathrm{H}_{29} \mathrm{O}_{15}$ & $\begin{array}{c}\mathrm{MS}^{2} \text { [677]: } 353.0879(100), 335.0806(21), \\
179.0342(21), 191.0555(13)\end{array}$ & 1,3,5-TriCQA \\
\hline 50 & 10.75 & 677.15119 & 677.15179 & 0.88 & $\mathrm{C}_{34} \mathrm{H}_{29} \mathrm{O}_{15}$ & $\begin{array}{c}\mathrm{MS}^{2}[677]: 353.0882(100), 179.0342(73) \\
161.0236(72), 515.1199(53), 335.0776(29) \\
173.0448(27), 497.1096(13), 191.0556(8)\end{array}$ & 1,3,4-TriCQA \\
\hline 51 & 11.20 & 497.10893 & 497.10956 & 1.72 & $\mathrm{C}_{25} \mathrm{H}_{21} \mathrm{O}_{11}$ & $\begin{array}{c}\mathrm{MS}^{2}[497]: 161.0235(100), 335.0775(60) \\
247.0797(36), 179.0342(32), 135.0440(20)\end{array}$ & DiCQL \\
\hline 52 & 11.26 & 499.12458 & 499.12451 & 1.02 & $\mathrm{C}_{25} \mathrm{H}_{23} \mathrm{O}_{11}$ & $\begin{array}{c}\mathrm{MS}^{2} \text { [499]: } 173.0447(100), 179.0342(42) \\
191.0554(29), 353.0878(9)\end{array}$ & 4C, 5-pCoQA \\
\hline 53 & 11.30 & 367.10346 & 367.10199 & -3.99 & $\mathrm{C}_{17} \mathrm{H}_{19} \mathrm{O}_{9}$ & $\begin{array}{c}\mathrm{MS}^{2} \text { [367]:173.0447 (100), } 134.03625(35), \\
191.0499(15)\end{array}$ & 4FQA \\
\hline 54 & 11.62 & 529.13515 & 529.13525 & 0.19 & $\mathrm{C}_{26} \mathrm{H}_{25} \mathrm{O}_{12}$ & $\begin{array}{c}\mathrm{MS}^{2}[529]: 173.0447(100), 179.0342(27) \\
191.0555(24), 193.0500(11)\end{array}$ & 4C, 5FQA \\
\hline 55 & 11.68 & 497.10893 & 497.10944 & 1.60 & $\mathrm{C}_{25} \mathrm{H}_{21} \mathrm{O}_{11}$ & $\begin{array}{c}\mathrm{MS}^{2}[497]: 161.0235(100), 179.0342(97) \\
135.0441(46), 247.0799(19), 119.0338(19) \\
\text { MS }^{2}[497]: 161.0235(100), 335.0775(56)\end{array}$ & DiCQL \\
\hline 56 & 12.24 & 497.10893 & 497.10950 & 1.66 & $\mathrm{C}_{25} \mathrm{H}_{21} \mathrm{O}_{11}$ & $\begin{array}{c}247.0797 \text { (35), } 179.0342(30), 137.0233(28), \\
135.0441(18)\end{array}$ & DiCQL \\
\hline 57 & 12.27 & 661.15628 & 661.15472 & -2.36 & $\mathrm{C}_{34} \mathrm{H}_{29} \mathrm{O}_{14}$ & $\begin{array}{c}\mathrm{MS}^{2}[661]: 353.0879(100), 337.0933(56) \\
191.0554(40), 179.0342(57), 173.0448(24) \\
161.0237(21), 163.0393(17)\end{array}$ & pCoDiCQA \\
\hline 58 & 12.28 & 691.16684 & 691.16840 & 2.25 & $\mathrm{C}_{35} \mathrm{H}_{31} \mathrm{O}_{15}$ & $\begin{array}{c}\mathrm{MS}^{2}[691]: 353.0879(100), 179.0343(75) \\
161.0236(55), 335.0776(29), 191.0553(24) \\
515.1195(22), 173.0448(21)\end{array}$ & DiCFQA \\
\hline
\end{tabular}


TABLE 1: Continued.

\begin{tabular}{|c|c|c|c|c|c|c|c|}
\hline Peak & $t_{R}$ & $\begin{array}{l}\text { Theoretical } \\
\text { mass }(\mathrm{m} / \mathrm{z})\end{array}$ & $\begin{array}{l}\text { Experimental } \\
\text { mass }(\mathrm{m} / \mathrm{z})\end{array}$ & $\begin{array}{l}\text { Error } \\
(\mathrm{ppm})\end{array}$ & $\begin{array}{c}\text { Formula } \\
{[\mathrm{M}-\mathrm{H}]}\end{array}$ & MS/MS fragment & $\begin{array}{l}\text { Identification/ } \\
\text { reactions }\end{array}$ \\
\hline 59 & 12.38 & 661.15628 & 661.15369 & -3.92 & $\mathrm{C}_{34} \mathrm{H}_{29} \mathrm{O}_{14}$ & $\begin{array}{c}\mathrm{MS}^{2}[661]: 337.0931(100), 353.0882(74), \\
173.0447(72), 179.0342(67), 191.0555(50), \\
335.0777(27), 499.1248(21), 515.1192(9)\end{array}$ & pCoDiCQA \\
\hline 60 & 12.56 & 691.16684 & 691.16821 & 1.98 & $\mathrm{C}_{35} \mathrm{H}_{31} \mathrm{O}_{15}$ & $\begin{array}{c}353.0880(69), 173.0448(42), 529.1351(32) \\
191.0554(24), 335.0775(17)\end{array}$ & DiCFQA \\
\hline 61 & 12.59 & 721.17741 & 721.17474 & -3.70 & $\mathrm{C}_{36} \mathrm{H}_{33} \mathrm{O}_{16}$ & $\begin{array}{c}\mathrm{MS}^{2}[721]: 329.1026(100), 353.0873(40) \\
515.1187(31), 173.0444(22), 179.0338(15) \\
191.0551(15), 335.0771(12), 161.0232(11) \\
151.0387(8)\end{array}$ & DiCSQA \\
\hline 62 & 12.72 & 677.15119 & 677.15173 & 0.79 & $\mathrm{C}_{34} \mathrm{H}_{29} \mathrm{O}_{15}$ & $\begin{array}{c}\mathrm{MS}^{2}[677]: 353.0879(100), 515.1198(69) \\
173.0447(26), 179.0341(22), 335.0777(12) \\
191.0557(7), 161.0233(6)\end{array}$ & 1,4,5-TriCQA \\
\hline 63 & 12.77 & 721.17741 & 721.17480 & -3.62 & $\mathrm{C}_{36} \mathrm{H}_{33} \mathrm{O}_{16}$ & $\begin{array}{c}\mathrm{MS}^{2}[721]: 353.0873(100), 191.0551(28) \\
173.0444(24), 179.0339(23), 559.1453(16) \\
329.1022(10), 335.0765(8), 515.1185(7) \\
161.0232(6)\end{array}$ & DiCSQA \\
\hline 64 & 13.07 & 721.17741 & 721.17267 & -6.57 & $\mathrm{C}_{36} \mathrm{H}_{33} \mathrm{O}_{16}$ & $\begin{array}{c}\left.\mathrm{MS}^{2} \text { [721]: } 353.0873(100), 173.0443451\right), \\
191.0550(31), 515.1193(30), 179.0339(26), \\
559.1444(18), 151.0387(17), 161.0444(16)\end{array}$ & DiCSQA \\
\hline 65 & 13.11 & 691.16684 & 691.16766 & 1.18 & $\mathrm{C}_{35} \mathrm{H}_{31} \mathrm{O}_{15}$ & $\begin{array}{c}\mathrm{MS}^{2}[691]: 529.1353(100), 367.1035 \text { (92), } \\
353.0879 \text { (74), } 173.0447(56), 179.0342(52), \\
335.0775 \text { (48), } 161.0235(17), 515.1199 \text { (11) }\end{array}$ & DiCFQA \\
\hline 66 & 13.28 & 721.17741 & 721.17426 & -4.37 & $\mathrm{C}_{36} \mathrm{H}_{33} \mathrm{O}_{16}$ & $\begin{array}{c}\mathrm{MS}^{2}[721]: 353.0873(100), 173.0444(20) \\
179.0338(13), 515.1189(12), 559.1451(11) \\
191.0550(9), 335.0769(4)\end{array}$ & DiCSQA \\
\hline 67 & 13.41 & 499.12458 & 499.12097 & -2.52 & $\mathrm{C}_{25} \mathrm{H}_{23} \mathrm{O}_{11}$ & $\mathrm{MS}^{2}$ [499]: 191.0554 (100), 173.0447 (46) & 3C, 5-pCoQA \\
\hline 68 & 13.45 & 721.17741 & 721.17163 & -8.01 & $\mathrm{C}_{36} \mathrm{H}_{33} \mathrm{O}_{16}$ & $\begin{array}{l}\mathrm{MS}^{2}[721]: 353.0874(100), 173.0443(25), \\
559.1452(19), 179.0338(14), 191.0552(13)\end{array}$ & DiCSQA \\
\hline
\end{tabular}

According the base peak and retention time, the absence of base peak at $\mathrm{m} / \mathrm{z} 173.044\left(\mathrm{C}_{7} \mathrm{H}_{9} \mathrm{O}_{5}\right)$ of compounds $36,37,43$, 45 , and 67 are consistent with their being 3C, 5pCoQA, Cis3-pCo, 5CQA, Cis-3-pCo, 5CQA, 3C, 5-pCoQA, and 3C, 5pCoQA. Besides, compound 52 was tentatively identified as 4C, 5-pCoQA $[15,19]$.

Compounds 27, 39, 40, and 42 were eluted at 6.08, 8.02, 8.31 , and $9.12 \mathrm{~min}$ and had the same quasimolecular ions $[\mathrm{M}-\mathrm{H}]^{-}$at $\mathrm{m} / \mathrm{z} 515.119\left(\mathrm{C}_{25} \mathrm{H}_{23} \mathrm{O}_{12}\right)$, possessed the same retention time and mass spectrum data with these reference standards 1,3-diCQA, 3,4-diCQA, 3,5-diCQA, and 4,5diCQA, respectively. Thus, they were unambiguously assigned as 1,3-diCQA, 3,4-diCQA, 3,5-diCQA, and 4,5diCQA $[20,21]$.

Compounds 21, 25, and 38 with the deprotonated ion $[\mathrm{M}-\mathrm{H}]^{--}$at $\mathrm{m} / \mathrm{z} 677.17285\left(0.78 \mathrm{ppm}, \mathrm{C}_{31} \mathrm{H}_{33} \mathrm{O}_{17}\right), 677.17468$ (3.48 ppm, $\left.\mathrm{C}_{31} \mathrm{H}_{33} \mathrm{O}_{17}\right)$, and 677.17145 (-1.30 ppm, $\left.\mathrm{C}_{31} \mathrm{H}_{33} \mathrm{O}_{17}\right)$, respectively, were eluted at 5.56, 5.88, and $7.38 \mathrm{~min}, 162 \mathrm{Da}\left(\mathrm{C}_{6} \mathrm{H}_{10} \mathrm{O}_{5}\right)$ more than diCQA, suggesting they were the hexoside of diCQA, which were further confirmed by the presence of fragment ions $\mathrm{m} / \mathrm{z} 515.117$ $\left(\mathrm{C}_{25} \mathrm{H}_{23} \mathrm{O}_{12}\right), 173.044\left(\mathrm{C}_{7} \mathrm{H}_{9} \mathrm{O}_{5}\right), 179.033\left(\mathrm{C}_{9} \mathrm{H}_{7} \mathrm{O}_{4}\right)$, and $191.054\left(\mathrm{C}_{7} \mathrm{H}_{11} \mathrm{O}_{6}\right)$. Therefore, they were inferred as diCQAhexoside [12-14, 17].

Compounds 17 and 20 were eluted at 4.87 and $5.34 \mathrm{~min}$, yielded the same deprotonated ion $[\mathrm{M}-\mathrm{H}]^{-}$at $\mathrm{m} / \mathrm{z} 839.225$ $\left(-3.62 \mathrm{ppm}, \mathrm{C}_{37} \mathrm{H}_{43} \mathrm{O}_{22}\right), 162 \mathrm{Da}\left(\mathrm{C}_{6} \mathrm{H}_{10} \mathrm{O}_{5}\right)$ more than
diCQA-hexoside, suggesting they were the dihexoside of diCQA. Therefore, they were tentatively identified as diCQA-dihexoside.

Compounds $35,44,46,47,48$, and 54 were eluted at 6.93, $9.54,9.76,10.11,10.28$, and $11.62 \mathrm{~min}$ and generated a quasimolecular ion $[\mathrm{M}-\mathrm{H}]^{-}$at $\mathrm{m} / \mathrm{z} 529.13611(1.82 \mathrm{ppm}$, $\mathrm{C}_{26} \mathrm{H}_{25} \mathrm{O}_{12}$ ), 529.13525 (0.19 ppm, $\left.\mathrm{C}_{26} \mathrm{H}_{25} \mathrm{O}_{12}\right), 529.13550$ (0.66 ppm, $\mathrm{C}_{26} \mathrm{H}_{25} \mathrm{O}_{12}$ ), 529.13550 (0.66 ppm, $\mathrm{C}_{26} \mathrm{H}_{25} \mathrm{O}_{12}$ ), 529.13550 (0.66 ppm, $\mathrm{C}_{26} \mathrm{H}_{25} \mathrm{O}_{12}$ ), and 529.13525 (0.19 ppm, $\left.\mathrm{C}_{26} \mathrm{H}_{25} \mathrm{O}_{12}\right)$. These fragment ions at $\mathrm{m} / \mathrm{z} 173.044\left(\mathrm{C}_{7} \mathrm{H}_{9} \mathrm{O}_{5}\right)$, $193.049\left(\mathrm{C}_{10} \mathrm{H}_{9} \mathrm{O}_{4}\right)$ or $353.086\left(\mathrm{C}_{16} \mathrm{H}_{17} \mathrm{O}_{9}\right)$, and 367 $\left(\mathrm{C}_{17} \mathrm{H}_{19} \mathrm{O}_{9}\right)$ were shown in all of those above compounds, which were consistent with caffeoylferuloylquinic acids (CFQA). Based on the retention time, data of $\mathrm{MS}^{2}$, and diagnosis ions in bibliography, compounds 35, 44, 46, 47, 48, and 54 were tentatively identified as 3C, 5FQA, 3F, 5CQA, 3F, 5CQA, 3F, 5CQA, 3C, 5FQA, and 4C, 5FQA, respectively $[16,20]$.

3.3. Identification of Triacyl-Quinic Acids or Triacyl-Shikimic Acids. Compounds 57 and 59 yielded a deprotonated ion $[\mathrm{M}-\mathrm{H}]^{-}$at $\mathrm{m} / \mathrm{z} 661.15472\left(-2.36 \mathrm{ppm}, \mathrm{C}_{34} \mathrm{H}_{29} \mathrm{O}_{14}\right)$ and 661.15369 (-3.92 ppm, $\left.\mathrm{C}_{34} \mathrm{H}_{29} \mathrm{O}_{14}\right)$ and were eluted at 12.27 and $12.38 \mathrm{~min}$, respectively. Based on the reference, retention time, fragment ions $\mathrm{m} / \mathrm{z} 173.044\left(\mathrm{C}_{7} \mathrm{H}_{9} \mathrm{O}_{5}\right), 179.033$ $\left(\mathrm{C}_{9} \mathrm{H}_{7} \mathrm{O}_{4}\right), 337.092\left(\mathrm{C}_{16} \mathrm{H}_{17} \mathrm{O}_{8}\right)$, and $353.087\left(\mathrm{C}_{16} \mathrm{H}_{17} \mathrm{O}_{9}\right)$ 


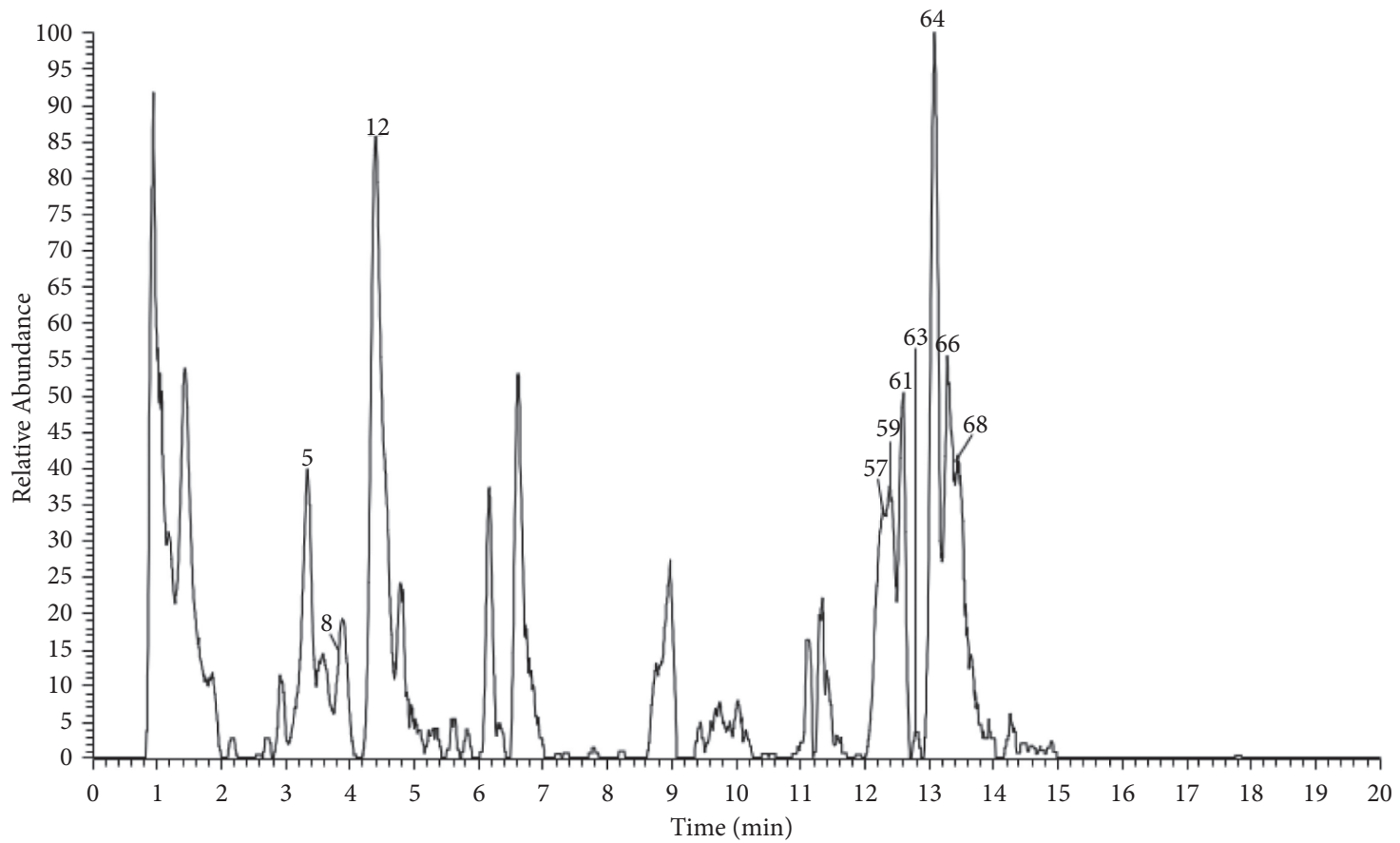

(a)

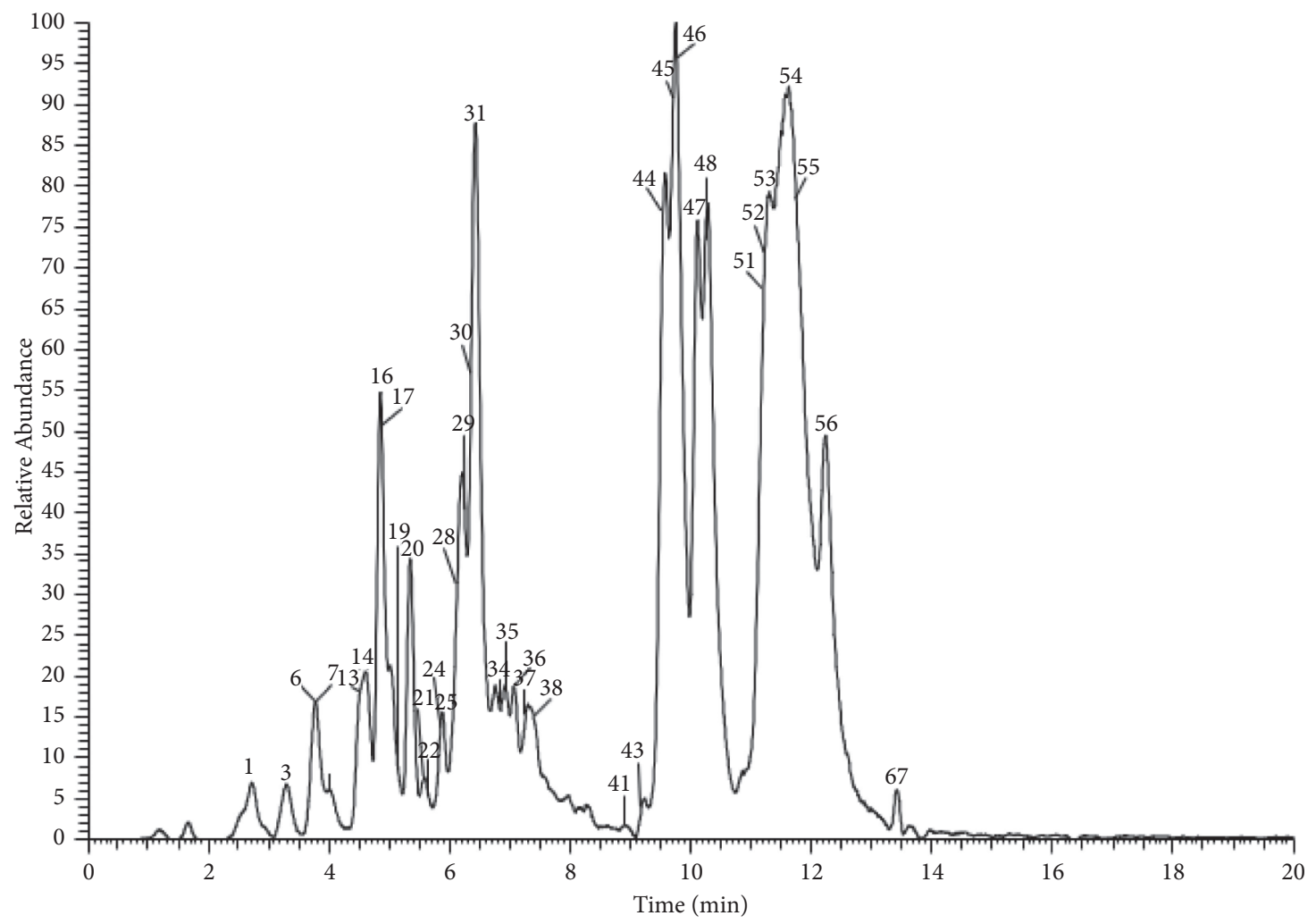

(b)

Figure 1: Continued. 


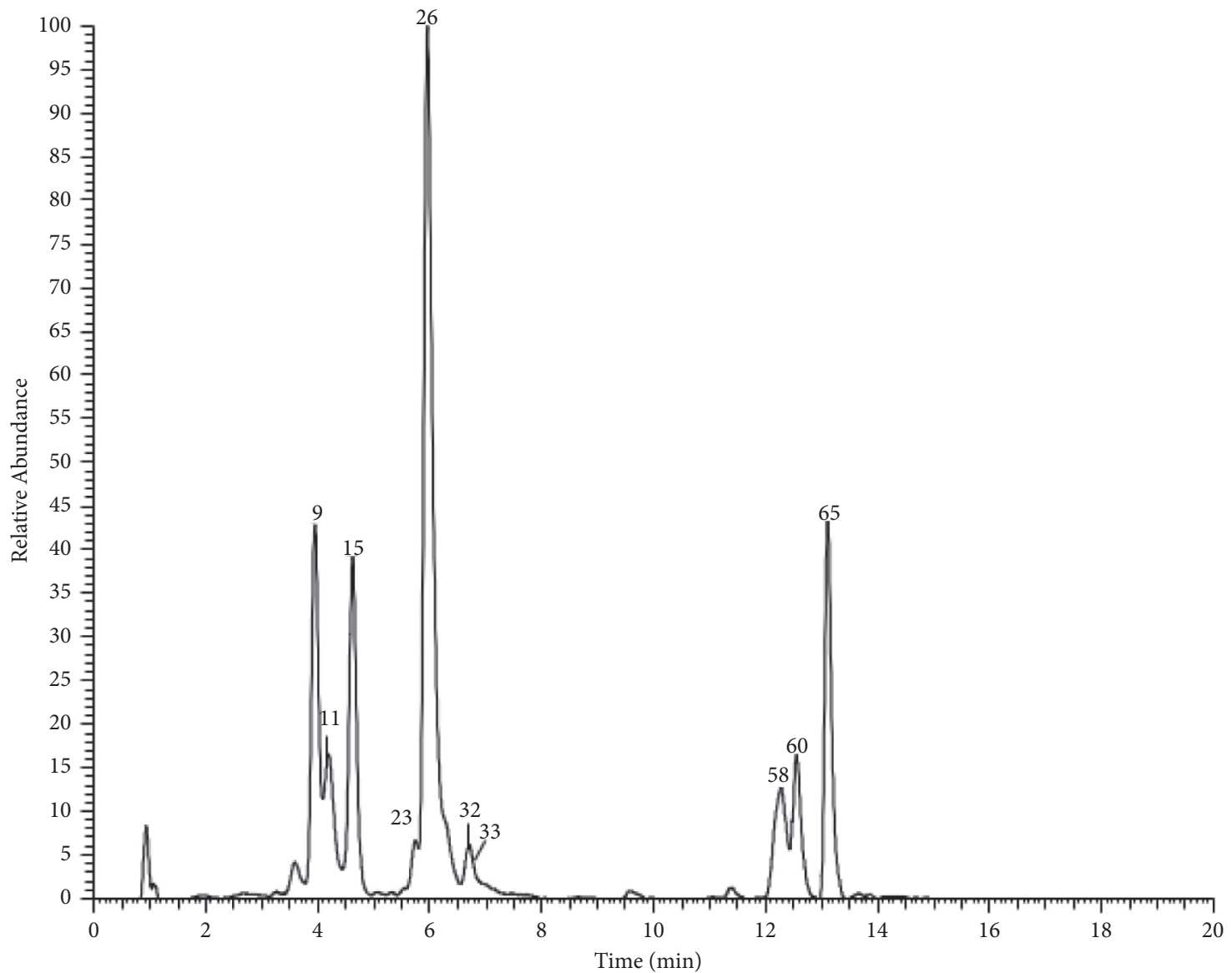

(c)

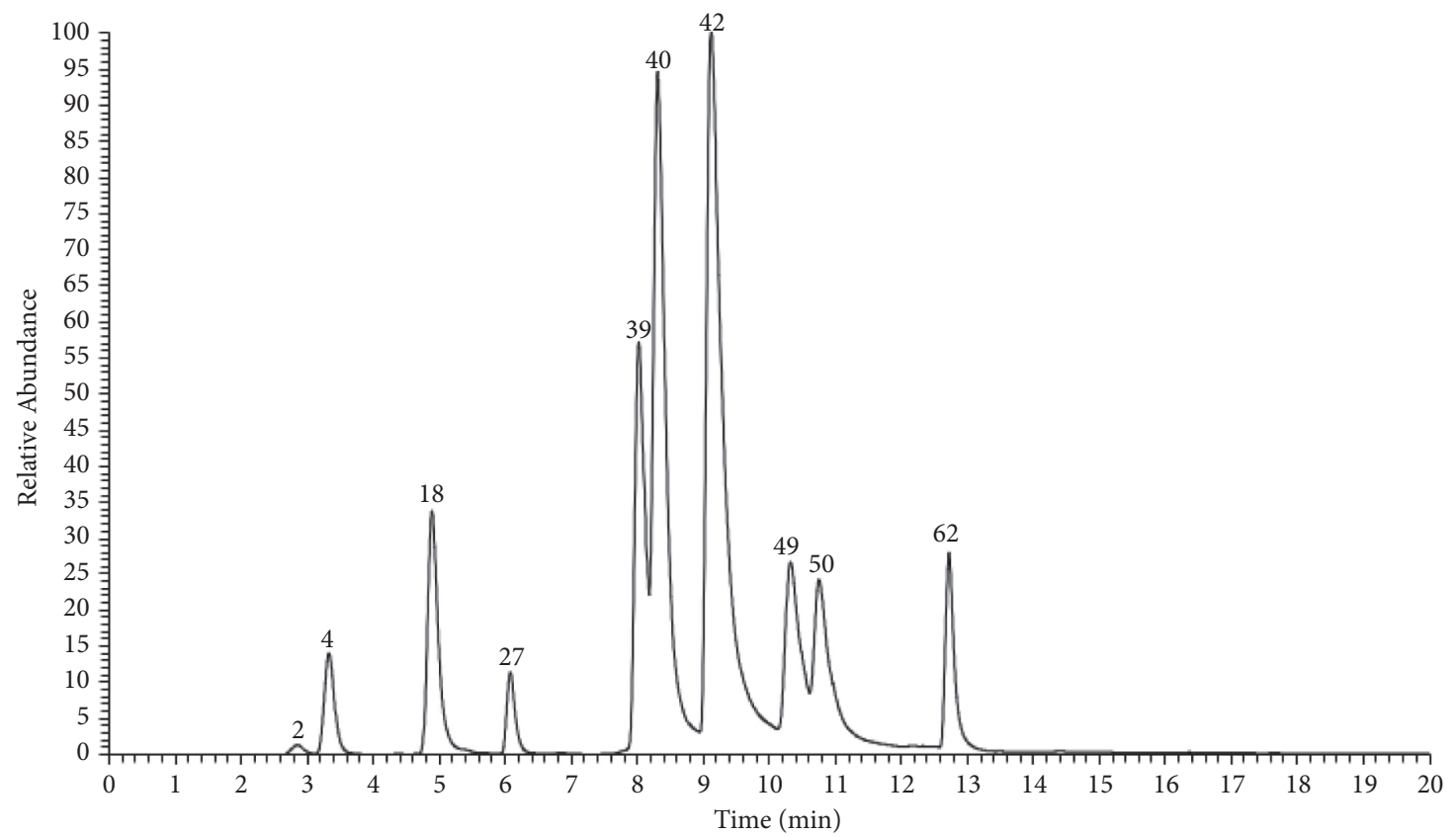

(d)

FIgURE 1: The high-resolution extracted ion chromatogram (HREIC) in 5 ppm for the multiple compounds in Inula cappa. (a) 353.10894, $529.15628,661.15628$, and 721.17741; (b) $335.07724,367.10346,497.10893,499.12458,499.14571,515.14063,529.13515,677.17232$, and 839.22515; (c) 337.09289, 341.08781, and 691.16684; (d) 353.08781, 515.1195, and 677.15119. 
indicated that they are $\mathrm{p}$-coumaroyl-dicaffeoylquinic acids (pCoDiCQA).

Compounds 49, 50, and 62 were eluted at 10.32, 10.75, and $12.72 \mathrm{~min}$, generated a quasimolecular ion $[\mathrm{M}-\mathrm{H}]^{-}$at $\mathrm{m} /$ z 677.15173 (0.75 ppm, $\left.\mathrm{C}_{34} \mathrm{H}_{29} \mathrm{O}_{15}\right), 677.15179$ (0.88 ppm, $\left.\mathrm{C}_{34} \mathrm{H}_{29} \mathrm{O}_{15}\right)$, and $677.15173\left(0.79 \mathrm{ppm}, \mathrm{C}_{34} \mathrm{H}_{29} \mathrm{O}_{15}\right)$, respectively. Based on the existence of fragment ions $\mathrm{m} / \mathrm{z}$ $353.0869\left(\mathrm{C}_{16} \mathrm{H}_{17} \mathrm{O}_{9}\right)$ and $\mathrm{m} / \mathrm{z} 515.1180\left(\mathrm{C}_{25} \mathrm{H}_{23} \mathrm{O}_{12}\right)$, the absence of ion $\mathrm{m} / \mathrm{z} 497.1070\left(\mathrm{C}_{25} \mathrm{H}_{21} \mathrm{O}_{11}\right)$ of compounds 49 , 50 , and 62 were tentatively identified as 1,3-C, 1,3,4-TriCQA and 1,4,5-TriCQA [20].

Compounds 58, 60, and 65 were eluted at 12.28, 12.56, and $13.11 \mathrm{~min}$, with a quasimolecular ion $[\mathrm{M}-\mathrm{H}]^{-}$at $\mathrm{m} / \mathrm{z}$ 691.16840 (2.25 ppm, $\left.\mathrm{C}_{35} \mathrm{H}_{31} \mathrm{O}_{15}\right), \quad 691.16821(1.98 \mathrm{ppm}$, $\mathrm{C}_{35} \mathrm{H}_{31} \mathrm{O}_{15}$ ), and 691.16766 (1.18 ppm, $\mathrm{C}_{35} \mathrm{H}_{31} \mathrm{O}_{15}$ ), respectively. The fragment ions $\mathrm{m} / \mathrm{z} 529.133\left(\mathrm{C}_{26} \mathrm{H}_{25} \mathrm{O}_{12}\right), 367.118$ $\left(\mathrm{C}_{21} \mathrm{H}_{19} \mathrm{O}_{6}\right), 173.044\left(\mathrm{C}_{7} \mathrm{H}_{9} \mathrm{O}_{5}\right)$, and $179.033\left(\mathrm{C}_{9} \mathrm{H}_{7} \mathrm{O}_{4}\right)$ were detected in $\mathrm{MS}^{2}$ data of those compounds, indicating that they were dicaffeoylferuloylquinic acids (DiCFQA).

According to the above principles, compounds 61, 63, 64, 66 , and 68 can be identified as dicaffeoyl-sinapoylquinic acid (DiCSQA).

3.4. Others. Compounds 9, 11, 15, and 33 were eluted at $3.97,4.16,4.64$, and $6.73 \mathrm{~min}$ and yielded a deprotonated ion $[\mathrm{M}-\mathrm{H}]^{-}$at $\mathrm{m} / \mathrm{z} 341.08798\left(1.27 \mathrm{ppm}, \mathrm{C}_{15} \mathrm{H}_{17} \mathrm{O}_{9}\right), 341.08801$ (1.30 ppm, $\mathrm{C}_{15} \mathrm{H}_{17} \mathrm{O}_{9}$ ), 341.08801 (1.30 ppm, $\mathrm{C}_{15} \mathrm{H}_{17} \mathrm{O}_{9}$ ), and $341.08786\left(0.16 \mathrm{ppm}, \mathrm{C}_{15} \mathrm{H}_{17} \mathrm{O}_{9}\right)$, which show a fragment ion at $\mathrm{m} / \mathrm{z} 179.033\left(\mathrm{C}_{9} \mathrm{H}_{7} \mathrm{O}_{4}\right)$ by losing the $162 \mathrm{Da}$ in the $\mathrm{MS}^{2}$ experiment. The fragment ions of $\mathrm{m} / \mathrm{z} 135.043\left(\mathrm{C}_{8} \mathrm{H}_{7} \mathrm{O}_{2}\right)$ and $179.033\left(\mathrm{C}_{9} \mathrm{H}_{7} \mathrm{O}_{4}\right)$ consisted with the caffeic acids; above all, they were tentatively identified as caffeoyl hexoside (CAhexoside) [22].

Compound 8 was detected at $3.83 \mathrm{~min}$ and generated the quasimolecular ion $[\mathrm{M}-\mathrm{H}]^{-}$at $\mathrm{m} / \mathrm{z} 353.10687$ (-0.97 ppm, $\left.\mathrm{C}_{13} \mathrm{H}_{21} \mathrm{O}_{11}\right)$. This compound yielded fragment ions at $\mathrm{m} / \mathrm{z}$ $191.055\left(\mathrm{C}_{7} \mathrm{H}_{11} \mathrm{O}_{6}\right)$ and $173.044\left(\mathrm{C}_{7} \mathrm{H}_{9} \mathrm{O}_{5}\right)$, and guess was composed of quinic acid fragments. Therefore, it might be considered as hexoside of quinic acid (QA-hexoside) [23].

\section{Conclusions}

In this study, 68 chlorogenic acid and derivatives exhaustively characterized from the extract of Inula cappa by using the strategy for the rapid detection and identification of CGAs using UHPLC-Q-Exactive Orbitrap mass spectrometry with diagnostic fragment ion technology was proposed. The developed strategy was proved reliable and efficient in rapid discovery of new CGA class from Inula cappa. The results will provide new insights into the effective substances and quality control of Inula cappa. At the same time, the yield of Inula cappa is very high, and the study of the chlorogenic acid can be used as a basis to judge whether it can be used as a substitute for endangered wild medicinal materials. Besides, it also provides new insights for understanding the qualitative characteristics of these phytochemical components in other TCM.

\section{Data Availability}

The data used to support the finding of this study are available from the corresponding author upon request.

\section{Conflicts of Interest}

The authors declare that they have no conflicts of interest.

\section{Authors' Contributions}

Jie Peng and Jing Xie contributed equally to this work.

\section{Acknowledgments}

This work was financially supported by the Natural Science Foundation of Hunan Province (2018JJ3376) and Hunan University of Medicine High-Level Talent Introduction Startup Funds (15001).

\section{References}

[1] F. Cheung, "Modern TCM: enter the clinic," Nature, vol. 480, no. 7378, pp. S94-S95, 2011.

[2] M. Noetzli, N. Ansermot, M. Dobrinas, and C. B. Eap, "Simultaneous determination of antidementia drugs in human plasma: procedure transfer from HPLC-MS to UPLC-MS/ MS," Journal of Pharmaceutical and Biomedical Analysis, vol. 64-65, pp. 16-25, 2012.

[3] J. Li, Y. Bai, P. Zhang et al., "Simultaneous determination of 5 flavonoids and 7 saponins for quality control of traditional Chinese medicine preparation xinnaoshutong capsule using HPLC-VWD-ELSD," Journal of Analytical Methods in Chemistry, vol. 2017, Article ID 3190185, 8 pages, 2017.

[4] T.-K. Ren, M.-L. Li, H. Zheng, Z. Wang, and J.-L. Zhang, "Characterization of acidic glycosphingolipid changes in C6 glioma rats treated with temozolomide using ultra-highperformance liquid chromatography coupled with quadrupole time-of-flight mass spectrometry," Journal of Analysis and Testing, vol. 4, no. 3, pp. 217-225, 2020.

[5] Jiangsu New Medical College, Dictionary of Chinese Materia Medica, Shanghai Science and Technology Publishing House, Shanghai, China, 1977.

[6] N. P. Long, S. Park, N. H. Anh et al., "Advances in liquid chromatography-mass spectrometry-based lipidomics: a look ahead," Journal of Analysis and Testing, vol. 4, no. 3, pp. 183-197, 2020.

[7] A. M. L. Seca, A. Grigore, D. C. G. A. Pinto, and A. M. S. Silva, "The genus inula and their metabolites: from ethnopharmacological to medicinal uses," Journal of Ethnopharmacology, vol. 154, no. 2, pp. 286-310, 2014.

[8] P. Matuschowski, A. Nahrstedt, and H. Winterhoff, "Pharmakologische untersuchungen eines frischpflanzenpresssaftes ausCynara scolymusauf choleretische Wirkung," Zeitschrift für Phytotherapie, vol. 26, no. 1, pp. 14-19, 2005.

[9] W. Cai, K.-L. Li, P. Xiong et al., "A systematic strategy for rapid identification of chlorogenic acids derivatives in Duhaldea nervosa using UHPLC-Q-exactive orbitrap mass spectrometry," Arabian Journal of Chemistry, vol. 13, no. 2, pp. 3751-3761, 2020.

[10] K. Masike, M. I. Mhlongo, S. P. Mudau et al., "Highlighting mass spectrometric fragmentation differences and similarities between hydroxycinnamoyl-quinic acids and 
hydroxycinnamoyl-isocitric acids," Chemistry Central Journal, vol. 11, no. 1, p. 29, 2017.

[11] H. Ouyang, J. Li, B. Wu et al., "A robust platform based on ultra-high performance liquid chromatography quadrupole time of flight tandem mass spectrometry with a two-step data mining strategy in the investigation, classification, and identification of chlorogenic acids in ainsliaea fragrans champ," Journal of Chromatography A, vol. 1502, pp. 38-50, 2017.

[12] R. Jaiswal, E. A. Halabi, M. G. E. Karar, and N. Kuhnert, "Identification and characterisation of the phenolics of Ilex glabra L. gray (aquifoliaceae) leaves by liquid chromatography tandem mass spectrometry," Phytochemistry, vol. 106, pp. 141-155, 2014.

[13] R. Jaiswal, M. F. Matei, P. Subedi, and N. Kuhnert, "Does roasted coffee contain chlorogenic acid lactones or/and cinnamoylshikimate esters?" Food Research International, vol. 61, pp. 214-227, 2014.

[14] R. Jaiswal, H. Müller, A. Müller, M. G. E. Karar, and N. Kuhnert, "Identification and characterization of chlorogenic acids, chlorogenic acid glycosides and flavonoids from Lonicera henryi L. (caprifoliaceae) leaves by LC-MS," Phytochemistry, vol. 108, pp. 252-263, 2014.

[15] R. Jaiswal, T. Sovdat, F. Vivan, and N. Kuhnert, "Profiling and characterization by LC-MSn of the chlorogenic acids and hydroxycinnamoylshikimate esters in maté (Ilex paraguariensis)," Journal of Agricultural and Food Chemistry, vol. 58, no. 9, pp. 5471-5484, 2010.

[16] M. N. Clifford, K. L. Johnston, S. Knight, and N. Kuhnert, "Hierarchical scheme for LC-MSn identification of chlorogenic acids," Journal of Agricultural and Food Chemistry, vol. 51, no. 10, pp. 2900-2911, 2003.

[17] M. N. Clifford, J. Kirkpatrick, N. Kuhnert, H. Roozendaal, and P. R. Salgado, "LC-MSn analysis of the cis isomers of chlorogenic acids," Food Chemistry, vol. 106, no. 1, pp. 379-385, 2008.

[18] M. N. Clifford, W. Wu, J. Kirkpatrick, and N. Kuhnert, "Profiling the chlorogenic acids and other caffeic acid derivatives of herbal chrysanthemum by LC-MSn," Journal of Agricultural and Food Chemistry, vol. 55, no. 3, pp. 929-936, 2007.

[19] M. N. Clifford, S. Marks, S. Knight, and N. Kuhnert, "Characterization by LC-MSn of four new classes of p-coumaric acid-containing diacyl chlorogenic acids in green coffee beans," Journal of Agricultural and Food Chemistry, vol. 54, no. 12, pp. 4095-4101, 2006.

[20] L. Liu, J. Zhang, B. Zheng et al., "Rapid characterization of chlorogenic acids in Duhaldea nervosa based on ultra-highperformance liquid chromatography-linear trap quadropoleorbitrap-mass spectrometry and mass spectral trees similarity filter technique," Journal of Separation Science, vol. 41, no. 8, pp. 1764-1774, 2018.

[21] M. N. Clifford, S. Knight, and N. Kuhnert, "Discriminating between the six isomers of dicaffeoylquinic acid by LC-MSn," Journal of Agricultural and Food Chemistry, vol. 53, no. 10, pp. 3821-3832, 2005.

[22] V. Gavrilova, M. Kajdžanoska, V. Gjamovski, and M. Stefova, "Separation, characterization and quantification of phenolic compounds in blueberries and red and black currants by HPLC-DAD-ESI-MSn," Journal of Agricultural and Food Chemistry, vol. 59, no. 8, pp. 4009-4018, 2011.

[23] J.-Y. Zhang, Z.-J. Wang, Y. Li et al., "A strategy for comprehensive identification of sequential constituents using ultra-high-performance liquid chromatography coupled with linear ion trap-orbitrap mass spectrometer, application study on chlorogenic acids in Flos lonicerae japonicae," Talanta, vol. 147, pp. 16-27, 2016. 